\title{
Differentiation between Autism and Multiple Complex Developmental Disorder in Response to Psychosocial Stress
}

\author{
Lucres MC Jansen*,1,2, Christine C Gispen-de Wied ${ }^{1,2}$, Rutger-Jan van der Gaag ${ }^{1,2}$ and Herman van \\ Engeland ${ }^{1,2}$ \\ 'Department of Child and Adolescent Psychiatry, University Medical Center, Utrecht, The Netherlands; ${ }^{2}$ RudolfMagnus Institute of Neuroscience, \\ Utrecht, The Netherlands
}

\begin{abstract}
Multiple Complex Developmental Disorder (MCDD) represents a distinct group within the autistic spectrum based on symptomatology. Unlike autistic children, part of MCDD children develop schizophrenia in adult life. Despite the differences, patients of both disorders are mainly characterized by abnormal reactions to their social environment. At the biological level, we showed in a previous study that MCDD children have a reduced cortisol response to psychosocial stress. Given the fact that autistic children clinically show more social impairments, it was hypothesized that they may have even further decreased cortisol responses to psychosocial stress than MCDD patients. Therefore, 10 autistic children were compared to 10 MCDD children and 12 healthy control children in their response to a psychosocial stressor, consisting of a public speaking task. In order to test whether any impairments in the biological stress response are specific for psychosocial stress, the autistic children were compared with II MCDD children and I5 control children in their response to a physical stressor, consisting of 10 min of bicycle exercise. Heart rate and salivary cortisol levels were used as indicators of response to the stress tests. Autistic children showed a relatively elevated cortisol response to psychosocial stress, in contrast to MCDD children who showed a reduced cortisol response. No differences in heart rate or cortisol responses to the physical stress test were found. The specific difference between autistic and MCDD children in their cortisol response to psychosocial stress indicates that the disturbed reactions to the social environment observed in these disorders may have different biological backgrounds.
\end{abstract}

Neuropsychopharmacology (2003) 28, 582-590. doi:I0.1038/sj.npp. 1300046

Keywords: psychosocial stress; exercise; cortisol; autism; MCDD

\section{INTRODUCTION}

Pervasive developmental disorders, or autistic spectrum disorders, are usually divided into autism and the autisticlike disorders. Of these, autism is the best described: the criteria have not changed much since the first description by Kanner (1943). However, ever since this first description, there has been discussion about the classification of children at the boundaries of classic autism. In DSM-IV, the autistic-like disorders are divided into Asperger's and Rett's disorder, for which clear diagnostic criteria are available, but most autistic-like children are placed in the rather ill-defined rest-group of Pervasive Developmental Disorders, Not Otherwise Specified (PDDNOS). In DSM-IV, this group is described as: 'This category should be used when there is a severe and pervasive impairment in the

*Correspondence: Dr LMC Nauta-Jansen, UMC Utrecht, Department of Child and Adolescent Psychiatry, HP F05. I26, PO Box 85500, 3508 GA Utrecht, The Netherlands, Tel: +31 30 2509729, Fax: +31 30 2505547, E-mail: L.M.C.Nauta-Jansen@psych.azu.nl

Received 17 April 2002; revised 31 July 2002; accepted 2 August 2002 Online publication: 6 August 2002 at http://www.acnp.org/citations/ Npp080602363 development of reciprocal social interaction or verbal and nonverbal communication skills, or when stereotyped behavior, interests, and activities are present, but the criteria are not met for a specific Pervasive Developmental Disorder, Schizophrenia, Schizotypal Personality Disorder, or Avoidant Personality Disorder.' (American Psychiatric Association, 1994). This lack of clear diagnostic criteria hampers research on this group of children. This is especially unfortunate, since the autistic-like disorders are even more common than autism itself. The ratio of the combined rate of all developmental disorders to the rate of autism is 2.6 (Fombonne, 1998).

Furthermore, the autistic-like disorders should not merely be regarded as some 'milder form' of autism. Several factor analyses on symptomatology have revealed that the autistic spectrum can be divided into four distinct groups on empirical grounds: besides the classical Kanner-type autistic patients, a group of Kanner-type autistic children with mental retardation, a group of atypical patients with more organic disturbances, and a group of children with more affective and 'schizoid' symptoms can be distinguished (Dahl et al, 1986; Siegel et al, 1986; Szatmari et al, 1989). The latter group matches the group of 'Multiple 
Complex Developmental Disorders' (MCDD), as described by Cohen et al (1986). MCDD children are different from autistic children in that they are more aggressive, more anxious, and show more psychotic thinking, suspiciousness and odd interactions than autistic children, while autistic children are more disturbed in their social interaction and communication and show more stereotyped and rigid behavior than MCDD children (Van der Gaag et al, 1995). Thus, MCDD children clearly differ from autistic children on symptomatological grounds. In 1994, Cohen et al (1994) established clear diagnostic criteria for this homogenous group of autistic-like children, which makes this a useful group for the research on autistic-like disorders (see the Appendix).

Another interesting indication that autistic-like children differ from autistic children, is the course of the disease. Follow-up studies show that autism is a relatively stable condition, while autistic-like disorders show a development towards the schizophrenic spectrum, with $6-29 \%$ of autistic-like children developing schizophrenia later in life. Similarly, $17 \%$ of MCDD children manifest schizophrenia as adults, while $56 \%$ develop a schizoid or schizotypal personality disorder (Van Engeland and Van der Gaag, 1994).

Despite these differences, abnormal reactions to the social environment and stress are still central to both disorders and have been described clinically. Autistic and autistic-like children can show hyper-reactions like tantrums, stereotyped behavior, and even self-injurious behavior. On the other hand, they can also show hyporeactions and appear unresponsive to their environment. One of the biological systems that play a major role in stress processing and adaptation to the environment is the hypothalamic-pituitary-adrenal (HPA) system. This system regulates the secretion of cortisol, the so-called stress hormone.
For MCDD children, we have previously found decreased cortisol stress responses to psychosocial stress (Jansen et al, 2000), and hypothesized that these children have an inability to interpret and elicit a normal biological response to their social environment. Thus, it may be hypothesized that autistic children, given the fact that they clinically show more social impairments, may have even further reduced cortisol responses to psychosocial stress. However, the literature on HPA function describes both normal and increased responses to stress in autism (Yamazaki et al, 1975; Maher et al, 1975; Tordjman et al, 1998). It is difficult to compare these studies with our own, since these studies used metabolic stress (Yamazaki et al, 1975; Maher et al, 1975), or a combined physical/psychological stressor like venapunction (Tordjman et al, 1998). Considering that the main impairments in autistic and MCDD children are found in their responses to the social environment, it would be more informative if both groups are compared in their responses to an actual psychosocial stress situation.

Therefore, in the first part of this study we compared the cortisol response to a psychosocial stressor in autistic children to our previous findings on the same psychosocial stressor in MCDD children. Then, to test if impairments in the HPA response are specific for psychosocial stress, in the second part of this study we compared the cortisol response to a physical stressor of the same autistic children with a group of MCDD children.

\section{SUBJECTS AND METHODS}

\section{Subjects}

Psychosocial stress subjects. In all, 10 autistic children were compared to 10 MCDD children and 12 healthy control children in their response to a psychosocial stressor. (see Table 1 for descriptive data of the study groups).

Table I Descriptive Data for All Study Groups

\begin{tabular}{|c|c|c|c|c|c|}
\hline & \multirow{2}{*}{$\begin{array}{l}\text { Autism } \\
\text { (Psychosocial+Physical) }\end{array}$} & \multicolumn{2}{|l|}{ MCDD } & \multicolumn{2}{|l|}{ Control } \\
\hline & & (Psychosocial) & (Physical) & (Psychosocial) & (Physical) \\
\hline Age & $9.4 \pm 1.4$ & $9.9 \pm 1.8$ & $9.4 \pm 1.7$ & $9.4 \pm 1.5$ & $10.0 \pm 2.0$ \\
\hline Male/female & $8 / 2$ & & & & $13 / 2$ \\
\hline IQ & $86(62-107)$ & $91(69-110)$ & $99(7 \mid-125)$ & & \\
\hline \multicolumn{6}{|l|}{$A D I$} \\
\hline Social interaction & $22.0 \pm 5.3$ & $|5.6 \pm 3 .|^{*} * *$ & & & \\
\hline Communication & $18.1 \pm 5.3$ & $9.7 \pm 2.9 * *$ & & & \\
\hline Stereotypical behavior & $7.4 \pm 3.5$ & $5.1 \pm 2.4$ & & & \\
\hline Onset & $3.2 \pm 1.8$ & $2.8 \pm 0.8$ & & & \\
\hline \multicolumn{6}{|l|}{ CBCL (T-scores) } \\
\hline Withdrawn & $65.9 \pm 8.2$ & $69.2 \pm 10.7$ & $62.7 \pm 12.0$ & $51.9 \pm 2.1$ & $51.7 \pm 4.5$ \\
\hline Somatic complaints & $54.7 \pm 6.6$ & $62.9 \pm 11.3$ & $61.6 \pm 8.8$ & $56.3 \pm 6.7$ & $55.5 \pm 6.3$ \\
\hline Anxious/depressed & $59.9 \pm 10.4$ & $72.8 \pm 11.8 *$ & $64.1 \pm 12.2$ & $54.0 \pm 7.0$ & $52.2 \pm 3.8$ \\
\hline Social problems & $71.7 \pm 11.7$ & $77.9 \pm 9.9$ & $70.1 \pm 11.8$ & $52.6 \pm 4.9$ & $52.3 \pm 3.5$ \\
\hline Thought problems & $74.8 \pm 6.4$ & $79.3 \pm 10.2$ & $69.0 \pm 9.8$ & $51.9 \pm 3.5$ & $51.6 \pm 4.6$ \\
\hline Attention problems & $71.7 \pm 10.0$ & $76.7 \pm 6.9$ & $71.9 \pm 9.5$ & $52.1 \pm 3.6$ & $54.3 \pm 5.0$ \\
\hline Delinquent behavior & $59.4 \pm 6.7$ & $63.9 \pm 12.2$ & $62.8 \pm 8.8$ & $54.0 \pm 6.1$ & $52.0 \pm 3.3$ \\
\hline Aggressive behavior & $60.3 \pm 7.7$ & $76.2 \pm 13.1 *$ & $70.0 \pm 7.4 *$ & $54.9 \pm 7.9$ & $52.0 \pm 5.1$ \\
\hline
\end{tabular}

Significant differences between MCDD and autistic groups are indicated with $* p<0.05$ and $* * *<0.01$. 
The MCDD and control children for the psychosocial stress test have been described in a previous report by our group (Jansen et al, 2000). The autistic and MCDD children were all recruited from the outpatients' clinic of the Department of Child Psychiatry of the University Medical Center Utrecht. Diagnoses were made according to DSM-IV criteria by extensive psychiatric evaluation consisting of a developmental history, medical examination, and semistructured psychiatric interview by experienced child psychiatrists (HvE, RJG). In addition, parents were interviewed using the Autism Diagnostic Interview (ADI) (LJ). The ADI gives impairment scores in the four main domains of the diagnostic criteria for autism: Qualitative Impairments in Social Interaction, Communication, Repetitive behaviors and Stereotyped Patterns, and Onset Evident before 36 months. For each domain, a cutoff score is defined above which the score falls within the autistic range. Cutoff scores are 10,8,3, and 1 , respectively for the four domains. MCDD children were all diagnosed as having PDDNOS according to DSM-IV. In addition, they all fulfilled the MCDD criteria as described by Cohen et al (1986) (see the Appendix).

Mean ADI scores for the autistic and MCDD children are given in Table 1. Although autistic children scored higher than MCDD children on all four domains, these differences only reached statistical significance in Qualitative Impairments in Social Interaction $(\mathrm{F}=9.89, p=0.006)$ and Communication $(\mathrm{F}=17.77, p=0.001)$. (It should also be noted that half of the MCDD children scored above the cutoff scores of the ADI. However, they did not fulfill the DSM-IV criteria for autism clinically. It is not known whether the ADI is overinclusive for the autistic-like disorders, because it has only been validated for autistic subjects in comparison with mentally handicapped and language-impaired subjects (Lord et al, 1994).)

Children with known mental retardation were excluded from the study, since all subjects should be able to understand and perform the public speaking task. Mean total IQs of the autistic and MCDD children, as assessed by either the Wechsler Intelligence Scale for Children-Revised NL (WISC-RN) (Wechsler, 1986) or the Kaufman Assessment Battery for Children (K-ABC) (Kaufman and Kaufman, 1983), were not significantly different (Table 1).

Physical stress subjects. The same group of 10 autistic children was compared to 10 MCDD children and 15 healthy controls in their response to a physical stressor (see Table 1 for descriptive data of the study groups).

The control children for the physical stress test have been described in a previous report by our group (Jansen et al, 1999), and the autistic children are described above. The MCDD children were again recruited from the outpatients' clinic of the Department of Child Psychiatry of the University Medical Center Utrecht and were diagnosed in the same way as the MCDD children used in the psychosocial stress test. Unfortunately, the parents of MCDD children could not be submitted to the ADI as it was not available in our language at the time of data collection (1995-1996). Total IQ scores were obtained in retrospect from the medical records for seven patients (see Table 1), and were not significantly different from the IQs of the MCDD and autistic groups used in the psychosocial stress test.

For all children, the Child Behavior Checklist (CBCL, Achenbach) was filled out by their parents. CBCL scores were used to screen control children for possible psychiatric problems. None of the children from the healthy control groups had any symptom cluster score above the 98th percentile. Secondly, CBCL scores were used to check for symptomatological differences like emotional problems, psychotic thinking, and aggressiveness not covered by the ADI, but known to differ between MCDD and autistic children (Van der Gaag et al, 1995). Indeed, MCDD children had higher scores than autistic children on anxiety/ depressed and the aggressive behavior cluster scores, but no differences were found on the thought problems cluster scores. Mean CBCL scores for all subject groups are given in Table 1. Main differences between the five groups were found on every CBCL score, except for somatic complaints. This was because of controls having significantly lower scores than patients. The two control groups did not differ from each other on any CBCL score. Analyses of differences between patient groups showed that the two MCDD groups differed on thought problems score (mean difference 10.33, $p=0.025$ ), but were similar to autistic patients on this score.

All children were screened for physical illness by filling out a medical checklist or by checking their medical records. Children with known endocrine, cardiovascular, pulmonary, liver or kidney diseases, or any organic cerebral disorders were excluded from participation in the study.

The study was approved by the Ethics Committee of the University Hospital Utrecht. Informed consent was obtained from parents and children.

\section{Stress Test Procedures}

Psychosocial stress test. This test consisted of speaking in public while being recorded on video. Children were asked to take part in a study on 'difficult tasks'. The exact content of the task was not explained, to avoid any anticipation for the public speaking task. After an initial resting period of $45 \mathrm{~min}$, the actual test began. It was not until then that the child was told that he/she had to perform a public speaking task. It was suggested that a 'jury' of at least three 'teachers' was present in the adjacent room behind a one-way screen, and that these 'teachers' would judge the child's performance on the task. The child was then given $10 \mathrm{~min}$ to prepare a 5-min talk on a subject of his/her own choice. During the talk, if the child stopped talking before the $5 \mathrm{~min}$ were over, a test assistant behind the one-way screen (one of the 'teachers') intervened via the intercom. The test assistant motivated the child to continue the talk, by saying: 'You have some time left, please continue', or 'Try to tell us something more about your subject'. After the talk, the child was left to wait for $5 \mathrm{~min}$ for the judgment of the 'teachers'. This judgment was always positive, thereby ending the stressful situation.

The public speaking test was imbedded in a 2-h stress test session, consisting of an initial resting period of $45 \mathrm{~min}$, the public speaking test, and a post-test resting period of $60 \mathrm{~min}$. The stress tests were compared to a control test that was identical to the stress test sessions, except that the 
subject did not have to perform the public speaking task. The resting periods and the control test session were spent as relaxed as possible, for instance by talking, drawing, or playing a game. Test sessions were performed on separate days between $10.00 \mathrm{am}$ and $4.00 \mathrm{pm}$, because the cortisol level is relatively stable and low during these hours, and is therefore more susceptible to stimulation. For each child the test sessions were performed at the same time, to rule out any differences in cortisol levels between the tests owing to circadian variation. Children were asked to refrain from eating and drinking and physical exercise for at least $1 \mathrm{~h}$ before the beginning of the test sessions.

Heart rate was measured continuously (Psylab data acquisition, Contact precision instruments) as a measure of autonomous activation. Saliva samples for the measurement of cortisol were collected at about 20-min intervals. The first saliva sample was taken during the initial resting period, 20 min before the start of the stress test. The second sample was taken just before the stress test. Then, saliva samples were taken after the preparation period and immediately after the 5-min talk. After the stress test, three more saliva samples were taken 20,40 , and $60 \mathrm{~min}$ after the end of the actual stress test (see also Table 2).

At the beginning of each test session, children used a visual analog scale (coloring a thermometer with a scale from 0 to 4 ) to score anticipation anxiety for the tests. Just before and after the stress tests, the Von Zerssen mood scale (Von Zerssen, 1986) (modified for children) was filled out to account for mood changes during the stress tests.

Video recordings of the public speaking task were used to score the performance of the public speaking task (observer). Parameters that were measured were the amount of time during the 5-min talk that the subject was actually speaking, and the number of interventions by the 'teachers' (test assistant) to motivate the child to continue the talk.

Physical stress test. This test consisted of $10 \mathrm{~min}$ of bicycle exercise on a home-trainer. The child was motivated to perform the test with maximum effort. Children were not informed about the content of the task until after the initial resting period, at the actual start of the exercise test, to rule out any anticipation stress.

The physical stress test was also imbedded in a 2-h stress test session similar to the psychosocial stress test session. Heart rate was measured automatically (Omron HEM705) as a measure of effort. Heart rate was assessed only before and after the actual exercise test, since data from control and MCDD children had already been collected between 1995 and 1996, and we had not been able to measure heart rate continuously at that time. Saliva samples for the measurement of cortisol were collected at about 20-min intervals, similar to the psychosocial stress test session, although sampling times differed slightly owing to the shorter duration of the physical stress test (see Table 3). Just as during the psychosocial stress test session, the anxiety thermometer and the Von Zerssen mood scale were filled out.

\section{Cortisol Analyses}

Saliva samples were collected in plastic vials after stimulation of saliva production with citric acid. Samples were stored at $-20^{\circ} \mathrm{C}$ until analysis. Saliva cortisol concentrations were measured without extraction, using an in-house competitive Radio-Immuno-Assay (RIA) with a polyclonal antibody to cortisol (K7348). [1,2- $\left.{ }^{3} \mathrm{H}(N)\right]$-Hydrocortisone (NET185, NEN-DUPONT, Dreieich, Germany) was used as a tracer after chromatographic verification of its purity. The lower limit of detection was $0.5 \mathrm{nmol} / 1$ and interassay variation was $11.0,8.2$, and $7.6 \%$ at $4.7,9.7$, and $14.0 \mathrm{nmol} / 1$, respectively $(n=20)$.

\section{Statistical Analyses}

Psychosocial stress test. Differences between autistic, MCDD, and control children in the effects of the psychosocial stress test on heart rate and cortisol levels

Table 2 Time Schedule for the Psychosocial Stress Test Session

\begin{tabular}{|c|c|c|c|c|c|c|c|}
\hline \multicolumn{2}{|c|}{ Initial resting period } & \multicolumn{3}{|c|}{ Psychosocial stress test } & \multicolumn{3}{|c|}{ Post-test resting period } \\
\hline$t=-45 \min$ & $t=-20$ & $\mathbf{t}=\mathbf{0}$ & $\mathbf{t}=\mathbf{1 5}$ & $\mathbf{t}=\mathbf{2 0}$ & $\mathbf{t}=\mathbf{4 0}$ & $t=60$ & $\mathbf{t}=\mathbf{8 0}$ \\
\hline \multicolumn{8}{|l|}{ Electrodes HR } \\
\hline & Saliva & Saliva & Saliva & Saliva & Saliva & Saliva & Saliva \\
\hline $\begin{array}{l}\text { Anxiety } \\
\text { thermometer }\end{array}$ & & $\begin{array}{l}\text { Von Zerssen } \\
\text { Instruction and } \\
\text { preparation }\end{array}$ & Public speaking & $\begin{array}{l}\text { Von Zerssen } \\
\text { Judgment } \\
\text { audience }\end{array}$ & & & \\
\hline
\end{tabular}

Table 3 Time Schedule for the Physical Stress Test Session

\begin{tabular}{|c|c|c|c|c|c|c|}
\hline \multicolumn{2}{|c|}{ Initial resting period } & \multicolumn{2}{|c|}{ Physical stress test } & \multicolumn{3}{|c|}{ Post-test resting period } \\
\hline$t=-45 \mathrm{~min}$ & $t=-20$ & $\boldsymbol{t}=\mathbf{0}$ & $t=10$ & $\mathbf{t}=\mathbf{3 0}$ & $\boldsymbol{t}=\mathbf{5 0}$ & $t=70$ \\
\hline $\begin{array}{l}\text { Anxiety } \\
\text { thermometer }\end{array}$ & Saliva & $\begin{array}{l}\text { Heart rate } \\
\text { Saliva } \\
\text { Von Zerssen } \\
\text { Bicycle exercise }\end{array}$ & $\begin{array}{l}\text { Heart rate } \\
\text { Saliva } \\
\text { Von Zerssen }\end{array}$ & Saliva & Saliva & Saliva \\
\hline
\end{tabular}


were tested by comparing these measures as obtained in the psychosocial stress test and control test, using repeated measures MANOVA, with test and time as the within-factors and diagnosis as the between-factor. Main effects of test by time and test by time by diagnosis were further analyzed by conducting difference contrast tests.

Differences in symptom scores, performance observation, anxiety thermometer, and Von Zerssen scores were tested using MANOVA. Pearson's correlation coefficients between psychometric scores and peak heart rate and cortisol levels after the psychosocial stress test were computed. If data were not normally distributed, the appropriate nonparametric tests were performed.

Physical stress test. Differences between autistic, MCDD, and control children in the effects of the physical stress test on heart rate and cortisol levels were tested using repeated measures MANOVA, with time as the within-factor and diagnosis as the between-factor. Main effects of time and time by diagnosis were further analyzed by conducting difference contrast tests.

Differences in symptom scores, anxiety thermometer, and Von Zerssen scores were tested by MANOVA. Pearson's correlation coefficients between psychometric scores and peak heart rate and cortisol levels after the physical stress test were computed. If data were not normally distributed, the appropriate nonparametric tests were performed.

\section{RESULTS}

\section{Psychosocial Stress Test}

Performance. All children in the study managed to give a talk during the public speaking test. The amount of time (in percent) that the subjects were actually speaking did not differ between diagnostic groups: mean amounts of time subjects were talking were $66.3 \pm 16.5 \%$ for control children, $65.6 \pm 12.9 \%$ for MCDD children, and $66.1 \pm 27.4 \%$ for the autistic children. The number of interruptions by the test assistant was also not significantly different between diagnostic groups, mean numbers of interruptions were $3.8 \pm 2.6$ for the control children, $9.1 \pm 9.8$ for the MCDD children, and $4.7 \pm 3.7$ for the autistic children. Performance on the public speaking test was not correlated with the patients' IQs.

Anxiety/mood. No differences were found in anxiety thermometer scores before the psychosocial and control tests. Furthermore, autistic, MCDD, and control children did not differ in their anxiety scores before any of the test sessions. MCDD children had higher Von Zerssen scores on all occasions, but after the control test $\left(\chi^{2}\right.$ values were resp. 6.63, $p<0.05$ before the control test; $4.88, p=0.09$ after the control test; 7.98, $p<0.05$ before the public speaking test, and 6.73, $p<0.05$ after the public speaking test) (see Table 4). Although all groups showed an increased Von Zerssen score after the psychosocial stress test, there was no overall significant increase in this score after the public speaking or the control tests.

Heart rate. Mean heart rates and salivary cortisol levels during the psychosocial and the control test for autism, MCDD, and healthy control children are given in Figure 1.

Repeated measures analysis revealed significant overall test by time effects for the heart rate $(\mathrm{F}=7.42, p<0.001)$. Difference contrasts showed that this effect was because of a significant increase during the preparation $(\mathrm{F}=5.57$, $p=0.03)$ and talk $(\mathrm{F}=17.86, p<0.001)$. Analyses of differences between autistic, MCDD, and control children revealed significant differences in the response to psychosocial stress (test by time by diagnosis effect: $\mathrm{F}=3.33$, $p=0.004)$. Difference contrasts revealed that the test by time by diagnosis effects were because of differences in the increase in heart rate during the preparation $(F=10.67$, $p<0.001)$, the talk $(\mathrm{F}=3.61, p=0.04)$, and the decrease after the talk $(\mathrm{F}=4.14, p=0.03)$.

Cortisol. Repeated measures analysis revealed significant overall test by time effects for salivary cortisol levels $(\mathrm{F}=7.97, p<0.001)$. Difference contrasts revealed that this was because of a significant increase during the preparation for the talk $(\mathrm{F}=6.19, p=0.02)$, the talk itself $(\mathrm{F}=17.46$, $p<0.001)$, and $20 \mathrm{~min}$ after the talk $(\mathrm{F}=11.28, p=0.002)$. Furthermore, a significant test by time by diagnosis effect was found $(\mathrm{F}=4.06, p=0.003)$. Difference contrasts revealed that this was because of significant differences during the talk $(\mathrm{F}=4.65, p=0.02), 20 \mathrm{~min}$ after the talk $(\mathrm{F}=5.97, p=0.007)$, and the decrease $40 \mathrm{~min}$ after the talk $(\mathrm{F}=3.73, p=0.04)$. Post hoc analyses on the cortisol concentrations during the public speaking and control test sessions revealed that MCDD children had significantly lower cortisol values as compared to healthy control children immediately after the public speaking test (contrast estimate $(\mathrm{CE})=-2.74, p=0.014$ ) and at 20 and $40 \mathrm{~min}$ after the test $(\mathrm{CE}=-5.13, p<0.001$ and $\mathrm{CE}=-2.82, p=0.001$ resp.). As for the cortisol levels during the control test, significantly higher levels were found for autistic children compared to healthy control children at the last three saliva sample times $(\mathrm{CE}=1.65$, $p=0.024 ; \mathrm{CE}=2.62, p=0.012$ and $\mathrm{CE}=2.52, p=0.011$ resp.) (see also Figure 1).

Table 4 Anxiety Thermometer and Von Zerssen Scores Psychosocial Stress

\begin{tabular}{|c|c|c|c|c|c|c|}
\hline & \multicolumn{2}{|c|}{ Anxiety thermometer } & \multicolumn{2}{|l|}{ Von Zerssen } & \multicolumn{2}{|l|}{ Von Zerssen } \\
\hline & Control test & Public speaking & Before control & After control & Before PS & After PS \\
\hline Autism & $0.85 \pm 1.06$ & $1.03 \pm 1.27$ & $1.50 \pm 2.5 \mid$ & $0.70 \pm 1.06$ & $0.60 \pm 1.07$ & $0.90 \pm 1.10$ \\
\hline MCDD & $0.72 \pm 1.19$ & $0.85 \pm 1.01$ & $2.90 \pm 2.42 *$ & $2.70 \pm 3.23$ & $3.00 \pm 2.54 *$ & $3.20 \pm 2.86 *$ \\
\hline Control & $0.64 \pm 0.88$ & $0.67 \pm 0.67$ & $0.67 \pm 1.37$ & $0.75 \pm 1.54$ & $0.92 \pm 1.08$ & $1.25 \pm 1.86$ \\
\hline
\end{tabular}

*Significantly different between groups. 
Correlations with symptom scores. ADI Communication scores appeared to be correlated with peak cortisol values at $t=40 \mathrm{~min}$ after the beginning of the public speaking test (Pearson's correlation coefficient $=0.515, p=0.029$ ), but it was not correlated with heart rate levels during the public speaking test. As for the CBCL symptom scores, anxious/ depressed symptoms and aggressive behavior were not correlated with peak cortisol values nor with heart rate levels during the public speaking task. In addition, there were no correlations between IQ scores and heart rate and cortisol responses to the public speaking test for the MCDD and autistic children (Pearson's correlation coefficients were resp. $-0.053, p=0.84$ and $0.013, p=0.962$ ).

\section{Physical Stress Test}

Heart rate. Mean heart rate and saliva cortisol levels during the physical stress test for autism, MCDD, and healthy control children are shown in Figure 2.

Repeated measures analysis revealed significant overall time effects for the heart rate before and after exercise $(\mathrm{F}=262.95, p<0.001)$, but no significant time by diagnosis effect $(\mathrm{F}=1.01, p=0.367)$.
Cortisol. Repeated measures analysis revealed a significant time effect $(\mathrm{F}=10.47, p<0.001)$ for salivary cortisol levels during the physical stress test. Difference contracts revealed that this was because of a significant rise in cortisol levels from 10 to $30 \mathrm{~min}$ after exercise $(\mathrm{F}=18.40, p<0.001)$. There was no significant time by diagnosis effect $(\mathrm{F}=2.12$, $p=0.101$ ) for the response to physical stress. Furthermore, an overall difference in cortisol levels was found between groups $(\mathrm{F}=3.41, p=0.046)$. Post hoc analyses revealed that control children had increased cortisol levels before the test at $t=-20(\mathrm{CE}=-2.67, p=0.003$ vs autistic children and $\mathrm{CE}=-3.08, p=0.001$ vs MCDD children) and at $t=0$ $(\mathrm{CE}=-2.03, p=0.021 v s$ autistic children and $\mathrm{CE}=-2.89$, $p=0.001 v s$ MCDD children). At $t=10$, control children only differed from MCDD children $(\mathrm{CE}=-2.45, p=0.004)$ (see also Figure 2).

Anxiety/mood. Autistic, MCDD, and control children had similar anxiety thermometer scores before the test. Scores in the Von Zerssen mood scale increased after the physical stress test $(Z=-2.73, p=0.006)$. In order to be able to compute differences in increase between the autistic, MCDD, and control group, Von Zerssen difference scores were computed. Analysis of these scores showed a


Figure I Mean heart rate and saliva cortisol curves for the psychosocial stress test session and the control test session for autistic, MCDD, and control children (--, autistic children, - $\mathbf{-}$-, MCDD children, - $\mathbf{\Delta}-$, control children).


Figure 2 Mean heart rate and saliva cortisol levels for the physical stress test session for autistic, MCDD, and control children (MCDD children, - $\mathbf{\Delta}-$, control children.) 
Table 5 Anxiety Thermometer and Von Zerssen Scores Physical Stress

\begin{tabular}{|c|c|c|c|c|c|c|}
\hline & \multicolumn{2}{|c|}{ Anxiety thermometer } & \multicolumn{2}{|l|}{ Von Zerssen } & \multicolumn{2}{|l|}{ Von Zerssen } \\
\hline & Control test & Exercise & Before control & After control & Before exercise & After exercise* \\
\hline Autism & $0.85 \pm 1.06$ & $0.69 \pm 1.31$ & $1.50 \pm 2.51$ & $0.70 \pm 1.06$ & $1.11 \pm 1.96$ & $0.67 \pm 0.87 *$ \\
\hline MCDD & $0.30 \pm 0.63$ & $0.61 \pm 1.10$ & $0.10 \pm 0.32$ & $0.30 \pm 0.48$ & $0.30 \pm 0.48$ & $3.00 \pm 2.83$ \\
\hline Control & $0.75 \pm 1.13$ & $1.09 \pm 1.13$ & $0.67 \pm 1.11$ & $0.73 \pm 1.58$ & $1.53 \pm 2.26$ & $3.00 \pm 2.56$ \\
\hline
\end{tabular}

significant difference in increase in Von Zerssen scores between autistic, MCDD, and control children after the physical stress test $\left(\chi^{2}=7.46, p=0.024\right)$. Contrast analyses revealed that this difference was owing to autistic children showing a smaller difference score $(Z=-2.20, p=0.028)$, while MCDD and control children did not differ from each other. Physical exercise had an impact on mood scores of MCDD and control children, but not on mood scores of autistic children (see Table 5).

Correlations with symptom scores. MCDD children that participated in the physical stress test scored significantly higher than autistic children did on aggressive behavior (mean difference score $-9.7, p=0.006$ ) (see also Table 1, note that these MCDD children comprised a different group than those tested in the psychosocial stress test), but there were no correlations between the heart rate or cortisol responses to the physical stress test and CBCL scores.

\section{DISCUSSION}

The main findings of the first part of the study were that autistic children did not differ from control children in their cortisol response to psychosocial stress, while their heart rate response to this test was significantly different from controls. In addition, autistic children showed an increase in saliva cortisol levels during the control test. In contrast, MCDD children showed both decreased heart rates and saliva cortisol responses to the psychosocial stress test, while their cortisol levels during the control test did not differ from those of controls.

The main finding of the second part of the study was that there were no differences between diagnostic groups for the heart rate and saliva cortisol response to physical stress. Thus, differences in heart rate and saliva cortisol stress responses between autistic, MCDD, and control children were specific for the reaction to psychosocial stress.

Autistic patients differed from control children in their psychosocial stress response in that they show normal cortisol responses in the face of decreased heart rate responses. During the actual talk, they showed a small but significant heart rate increase, indicating that they perceived the test as stressful in some way. However, they did not show an increase in heart rate in anticipation of the talk, during the preparation period, unlike normal control children. This may indicate some sort of inability to understand and anticipate the stressful social situation. The normal cortisol response, given the inability of autistic children to fully perceive the social stress situation, may be interpreted as a hyper-responsivity of the HPA system. Similarly, the increase in cortisol levels during the control test may be interpreted as an HPA hyper-response to a situation that is not perceived as stressful. This finding of an HPA hyperresponsiveness is consistent with earlier reports of increased HPA responsivity to stress in autistic patients (Tordjman et al, 1998; Maher et al, 1975). On the other hand, the increase in cortisol levels during the control test may also be interpreted as a disturbance of the circadian rhythm of the HPA axis, as has been previously found in autistic subjects (Yamazaki et al, 1975; Hill et al, 1977; Hoshino et al, 1987). Although this may not explain the initial cortisol response after the public speaking test, since this response occurred at least $20 \mathrm{~min}$ earlier, it may have influenced the duration of the response after the public speaking test.

The results of the second part of this study confirm the hypothesis that the impairments in stress responsivity are indeed specific for the reaction to psychosocial stress, since all children showed normal responses to the physical stress test. However, the finding of a normal cortisol response to physical stress in this group of MCDD children is inconsistent with our previous finding of a blunted cortisol response to physical stress in a sample of PDDNOS patients, who almost all fulfilled the MCDD criteria (Jansen et al, 1999). This difference might be explained by the fact that the PDDNOS patients in the previous study were all hospitalized, while the children in this study were all outpatients. Hospitalized children may be more severely ill, and may therefore have more severe HPA dysfunctions. However, comparison of symptom scores did not confirm this hypothesis (unpublished results). It should also be noted that the two MCDD groups used in the present study showed some symptomatological differences. There was a significant difference in CBCL scores for thought disorders between the two MCDD groups. This suggests that the MCDD group maybe less homogeneous than expected from the literature. It is not known whether the groups also differ in specific autistic-like symptoms, since unfortunately we did not have ADI scores of both MCDD groups.

The most interesting finding of this study is the fact that autistic and MCDD children differed considerably from each other in their reactions to psychosocial stress. Both disorders are characterized by an impairment in social functioning and by the inability to correctly interpret, and react to, their social environment. Therefore, based on our findings in MCDD children, and the fact that autistic children show even more social impairments, we had expected autistic children to have even more diminished cortisol responses to psychosocial stress. However, autistic children did not show decreased, but relatively increased cortisol responses to psychosocial stress.

Although at a symptomatological level, autistic children indeed differed significantly from MCDD children, this 
could not explain the differences in cortisol response to the public speaking test. In fact, the only symptom score that was significantly correlated to the cortisol response to psychosocial stress was a higher communication score on the ADI. However, this was a positive correlation, meaning that more impairments were related to higher cortisol levels. This is in contrast to our expectation that disturbances in social functioning would be related to diminished cortisol responses. Also, IQ appeared not to be an explaining factor for the differing response to public speaking between autistic and MCDD children. There was no significant correlation between IQ scores and heart rate or cortisol response to the psychosocial stress test. Unfortunately, we did not have IQ data of the control children and it is therefore not possible to determine if IQ would be a factor in the cortisol response in these children. However, the fact that autistic and MCDD children did not differ in IQ but showed completely different cortisol responses suggests that it is unlikely that IQ is an explaining factor for this difference.

Obviously, the processes behind abnormal reactions to psychosocial stress are far more complicated and are not easily explained by differences in symptomatology or overt behavior. Instead, the difference between autistic and MCDD children in their responses to psychosocial stress may indicate that the abnormal reactions to the social environment and stress as described clinically in autism and MCDD may have different biological backgrounds. Our measurement of only the end product of the biological stress response, cortisol release, is not sufficient to be able to say anything about the central mechanisms involved in (disturbances of) the psychosocial stress response. It is, therefore, extremely difficult to find proper explanations for the presently found differences between autism and MCDD, having used a neuroendocrine strategy only. The current findings warrant further research on the biological mechanisms that regulate the reaction to psychosocial stress and activation of the HPA axis. Important brain structures for cognitive stress processing, interpretation of stimuli, and triggering of the HPA and autonomous system are the prefrontal cortex and limbic structures like the amygdala (Huether, 1996). In neuroanatomical studies, reduced limbic structure volumes have been found to be correlated to autism (Bauman and Kemper, 1985; Jacobson et al, 1988). The authors have so far not found any specific neuroanatomical studies on autistice-like disorders. Furthermore, the study of the neuropeptides arginin-vasopressin (AVP) and oxytocin (OT) may be of interest. Both neuropeptides are involved in the regulation of the HPA response in reaction to stress, and psychological stress in particular (Romero and Sapolsky, 1996). AVP stimulates the HPA response, while OT is thought to inhibit the HPA response. Moreover, AVP and OT both play an essential role in the stimulation of social behaviors that are genetically determined (Insel, 1997; Dantzer et al, 1987). Thus, it has been hypothesized that abnormalities in AVP and OT may play an important role in disorders like autism, in which disturbances in social behavior are the main characteristics (Insel et al, 1999). Indeed abnormal basal concentrations of AVP and OT have been found in autistic children: basal AVP concentrations were found to be increased (Leboyer et $a l, 1992)$ and OT concentrations were found to be decreased
(Modahl et al, 1998). Thus, the elevated cortisol response to psychosocial stress might be explained by increased stimulation by AVP and decreased inhibition by OT. Similarly, the reduced cortisol response in MCDD children may be related to decreased stimulation by AVP and/or increased inhibition by OT. Finally, the selective impairment in the response to psychosocial stress that is found in MCDD children has also been found in schizophrenic patients (Jansen et al, 2000b). Part of MCDD children develop schizophrenia in adult life. Therefore, the impaired response to psychosocial stress in MCDD children may be a factor in the vulnerability to develop schizophrenia in later life, which is not present in autistic children. It might even be argued that MCDD children should be placed in the schizophrenic spectrum, rather than the autistic spectrum, as has previously been proposed for Multidimensionally Impaired Disorder, whose criteria largely overlap those for MCDD (Kumra et al, 1998).

Further research on the regulation of the response to psychosocial stress in autism, MCDD, and even schizophrenia is needed to unravel and interpret the differences found in the present study. For this, new strategies for investigating psychoneuroendocrinological systems should be addressed, in combination with both structural and functional imaging techniques (such as functional MRI and PET), as well as genetic studies.

\section{REFERENCES}

American Psychiatric Association (1994). Diagnostic and Statistical Manual of Mental Disorders, 4th edn DSM-IV. Washington, DC: APA.

Bauman M, Kemper TL (1985). Histoanatomic observations of the brain in early infantile autism. Neurology 35: 866-874.

Cohen DJ, Paul R, Volkmar FR (1986). Issues in the classification of Pervasive and Other Developmental Disorders: toward DSMIV. J Am Aca Child Psychiatry 25: 213-220.

Cohen DJ, Towbin KE, Mayes L, Volkmar F (1994). Developmental psychopathology of multiplex developmental disorder. In: Friedman SL, Haywood HC (eds). Developmental Follow-up: Concepts, Genres, Domains, and Methods. Academic Press: San Diego.

Dahl EK, Cohen DJ, Provence S (1986). Clinical and multivariate approaches to the nosology of pervasive developmental disorders. J Am Acad of Child Psychiatry 25: 170-180.

Dantzer R, Bluthe R-M, Koob GF, Le Moal M (1987). Modulation of social memory in male rats by neurohypophyseal peptides. Psychopharmacology (Berl) 91: 363-368.

Fombonne E (1998). Epidemiological surveys of autism. In: Volkmar FR (ed). Autism and Pervasive Developmental Disorders. Cambridge University Press: Cambridge. pp 32-63.

Hill SD, Wagner EA, Shedlarski Jr JG, Sears SP (1977). Diurnal cortisol and temperature variation of normal and autistic children. Dev Psychobiol 10: 579-583.

Hoshino Y, Yokoyama F, Watanabe M, Murata S, Kaneko M, Kumashiro $H$ (1987). The diurnal variation and response to dexamethasone suppression test of saliva cortisol level in autistic children. Jpn J Psychiatry Neurol 41: 228-235.

Huether G (1996). The central adaptation syndrome: psychosocial stress as a trigger for adaptive modifications of brain structure and brain function. Prog Neurobiol 48: 569-612.

Insel TR (1997). A neurobiological basis of social attachment. Am J Psychiatry 154: 726-735. 
Insel TR, O’Brien D, Leckman J (1999). Oxytocin, vasopressin, and autism: is there a connection? Biol Psychiatry 45: 145-157.

Jacobson R, Le Couteur A, Howlin P, Rutter M (1988). Selective subcortical abnormalities in autism. Psychol Med 18: 39-48.

Jansen LMC, Gispen-de Wied CC, Jansen MA, Van der Gaag RJ, Matthys M, Van Engeland H (1999). Pituitary-adrenal reactivity in a child psychiatric population: salivary cortisol response to stressors. Eur Neuropsychopharmacol 9: 67-75.

Jansen LMC, Gispen-de Wied CC, Van der Gaag RJ, Ten Hove F, Willemsen-Swinkels SHN, Harteveld E et al (2000). Unresponsiveness to psychosocial stress in a subgroup of autistic-like children, multiple complex developmental disorder. Psychoneuroendocrinology 25: 753-764.

Jansen LMC, Gispen-de Wied CC, Kahn RS (2000b). Selective impairments in the stress response in schizophrenic patients. Psychopharmacology 149: 319-325.

Kanner L (1943). Autistic disturbances of affective contact. Nerv Child 2: 217-250.

Kaufman AS, Kaufman NL (1983). Kaufman Assessment Battery for Children. American Guidance Service: Minnesota.

Kumra S, Jacobsen LK, Lenane M, Zahn TP, Wiggs E, Alaghband Rad J et al (1998). 'Multidimensionally impaired disorder': is it a variant of very early-onset schizophrenia? J Am Acad Child Adolesc Psychiatry 37: 91-99.

Leboyer M, Bouvard MP, Launay J-M, Tabutau F, Waller D, Dugas M et al (1992). Brief report: a double-blind study of naltrexone in infantile autism. J Autism Dev Disord 22: 309-319.

Lord C, Rutter M, Le Couteur A (1994). Autism diagnostic interview-revised - a revised version of a diagnostic interview for caregivers of individuals with possible pervasive developmental disorders. J Autism Dev Disord 24: 659-685.

Maher KR, Harper JF, Macleay A, King MG (1975). Peculiarities in the endocrine respons to insulin stress in early infantile autism. $J$ Nerv Ment Dis 161: 180-184.
Modahl C, Green L, Fein D, Morris M, Waterhouse L, Feinstein C et al (1998). Plasma oxytocin levels in autistic children. Biol Psychiatry 43: 270-277.

Romero LM, Sapolsky RM (1996). Patterns of ACTH secretagog secretion in response to psychological stimuli. J Neuroendocrinol 8: $243-258$.

Siegel B, Anders TF, Ciaranello RD, Bienenstock B, Kraemer HC (1986). Empirically derived subclassification of the autistic syndrome. J Autism Dev Disord 16: 275-293.

Szatmari P, Bartolucci G, Bremner R (1989). Asperger's syndrome and autism: comparison of early history and outcome. Dev Med Child Neurol 31: 709-720.

Tordjman S, McBride PA, Hertzig ME, Snow ME, Thompson SM, Anderson GM et al (1998). Plasma beta-endorphin, adrenocorticotropin hormone, and cortisol in autism. J Child Psychol Psychiatry 38: 705-715.

Van der Gaag RJ, Buitelaar JK, Van den Ban E, Bezemer M, Njio L, Van Engeland H (1995). A controlled multivariate chart review of multiple complex developmental disorder. $J \mathrm{Am}$ Acad Child Psychiatry 34: 1096-1106.

Van Engeland H, Van der Gaag RJ (1994). MCDD in childhood: a precursor of schizophrenic spectrum disorders. Schizophr Res 11: 197-197.

Von Zerssen D (1986). Clinical self-ratings scales (CSRS) of the Munich Psychiatric Information System. In: Sartorius $\mathrm{N}$, Ban TA (eds). Assessment of Depression. Springer-Verlag: Berlin. pp 270-303.

Wechsler D (1986). Wechsler Intelligence Scale for Children- revised NL. Swets \& Zeitlinger: Lisse.

Yamazaki K, Saito Y, Okada F, Fujieda T, Yamashita I (1975). An application of neuroendocrinological studies in autistic children and heller's syndrome. J Autism Child Schizophr 5: 323-332.

\section{APPENDIX 1}

The following gives diagnostic criteria for Multiple Complex Developmental Disorder.

(1) Regulation of affective state and anxiety is impaired beyond that seen in children of comparable age, as exemplified by at least two of the following:

(a) intense generalized anxiety or tension

(b) fears and phobias (often unusual or peculiar)

(c) recurrent panic episodes or 'flooding' with anxiety

(d) episodes of behavioral disorganization punctuated by markedly immature, primitive, or violent behaviors

(e) significant and wide emotional variability with or without environmental precipitants

(f) frequent idiosyncratic or bizarre anxiety reactions

(2) Consistently impaired social behavior/sensitivity, as exemplified by at least two of the following:

(a) social disinterest, detachment, avoidance, or withdrawal despite evident competence

(b) severely impaired peer relationships

(c) markedly disturbed attachments; high degrees of ambivalence to adults (esp. parents/caretakers)

(d) profound limitations in the capacity for empathy or understanding others affects accurately

(3) Impaired cognitive processing (thinking disorder) beyond that seen in children of comparable age, as exemplified by at least two of the following:

(a) irrationality, sudden intrusions on normal thought process, magical thinking, neologisms or repetition of nonsense words, desultory thinking, blatantly illogical, bizarre ideas

(b) confusion between reality and inner fantasy life

(c) perplexity and easy confusability (trouble understanding social processes or keeping thoughts 'straight')

(d) delusions, overvalued ideas including fantasies of omnipotence, paranoid preoccupations, overengagement with fantasy figures, grandiose fantasies of special powers, and referential ideation

(4) The child is not suffering from autism

(5) Duration of symptoms for at least 6 months

(reproduced from Cohen et al, 1994) 